\title{
Non pharmaceutical approach to COVID-19: The chemical science intervention
}

\author{
Sulaiman Ayodeji Apampa 1, ${ }^{*}$, Aisha Y. Lawal ${ }^{1}$ and David Adeniran Adedapo ${ }^{2}$ \\ ${ }^{1}$ Department of Chemistry, Federal College of Education, Zaria, Kaduna State, Nigeria. \\ ${ }^{2}$ Department of Chemistry, Federal College of Education, Kano, Kano State, Nigeria.
}

GSC Biological and Pharmaceutical Sciences, 2022, 18(03), 016-022

Publication history: Received on 26 January 2022; revised on 28 February 2022; accepted on 02 March 2022

Article DOI: https://doi.org/10.30574/gscbps.2022.18.3.0093

\begin{abstract}
COVID-19 pandemic issues are discussed in chemical science intuition which highlights the direct role of chemistry to the ongoing global pandemic by covering chemical preventive measures. Chemical preventive measures such as soap, water, hand sanitizer, disinfectants and personal protection equipment (PPE) were reviewed as the only available options in the arsenal to fight against COVID-19 despite the availability of COVID - 19 vaccines. The present review focused on the mode of action for both sanitizer and soap water to inactivate the virus and their effectiveness.
\end{abstract}

Keywords: Non pharmaceutical approach; COVID-19; Personal protective equipment's; Chemical Science Intervention.

\section{Introduction}

COVID-19 (Corona Virus Disease 2019) is an infectious disease caused by the novel severe acute respiratory syndromecoronavirus-2 (SARS-CoV-2). It has developed into a global pandemic rapidly disrupting modern society on a large scale that most of the people in the world have never witnessed in their lifetime [1] [2] [3] [4]. It was believed that this virus had its origin in a species of bat and it was transmitted to human via a host. Since then, human to human rapid spreading has been observed [5]. This novel corona virus is very close to earlier reported coronaviruses SARS-CoV-1, MERS-CoV (Middle East respiratory syndrome) with regard to causing severe acute respiratory distress [6]. The virus causes severe disease with pneumonia and acute respiratory distress and disorder, and has already infected millions of people, resulting in millions of deaths and the figures are continuously increasing with people's lives at risk and the global economy being greatly affected.

In order to address the current global challenge, public healthcare workers are working at the forefront to reduce the spread of this disease. With immense effort, rapid developments have been done by biologists and biomedical scientists to understand the biological responses against this viral infection, including detection of the virus, gene sequencing and determining protein structures [1] [7] [8]. Personal preventive measures including chemically made protective measures such as soap water, bleach solution, alcohol-based hand sanitizers, hydrogen peroxide and personal protective equipment have been conceptualized, prepared and in use to attack the viruses [9].

A novel coronavirus strain linked with fatal respiratory illness was reported in late 2019 [1]. Swift actions were taken by the Centre for Disease Control and Prevention (CDC), Chinese health authorities, and researchers. The World Health Organization (WHO) temporarily named this pathogen 2019 novel coronavirus (2019-nCoV) [2].

\footnotetext{
${ }^{*}$ Corresponding author: Sulaiman Ayodeji Apampa

Department of Chemistry, Federal College of Education, Zaria, Kaduna State, Nigeria. 


\section{Non-pharmaceutical chemical preventive measures}

Personal preventive measures are playing a vital role in stopping the spread of the SARS-CoV-2 virus (COVID 2019 virus) in the absence of any medicine or drug. Generally, these preventive measures can be physical, chemical or immunological in nature. For instance, spreading can be regulated by following social distancing of one meter at least and using face mask which comes under physical preventive measures. Chemical preventive measures include disinfecting surfaces using chemicals, washing hands with soap water or hand sanitization. Herd immunity, developed in the person who already recovered from the COVID-19 or who acquired vaccine, can break the chain of infection in a population [10]. Even with the development of vaccine, it is very essential for us to follow personal protective measures strictly to avoid infection from SARS-CoV-2. Well known non-pharmaceutical chemical preventive measures recommended for use against contracting the virus have been specified and documented [10].

The non-pharmaceutical chemical preventive measures are into two categories, namely the personal protective equipment which comprises of face masks, protective jackets and hand gloves. The second categories are chemically based substances which include soaps and detergents, hand sanitizers, bleach and hydrogen peroxide.

\subsection{Personal Protective Equipment (PPE)}

Personal protective equipment (PPE) used or that may be worn in the provision of healthcare includes gloves, aprons, protective jackets or long sleeved gowns, goggles, fluid-repellant surgical masks, face visors and respirator masks which create a barrier between healthcare workers and an infectious agent from the patient and to reduce the risk of transmitting micro-organisms such as SARS-CoV-2 (COVID -19 Virus) from healthcare workers to patient(s) or vice versa. In addition, personal protective equipment may sometimes be used by the patient's family/visitors, particularly if they are providing direct patient care such as assisting the patient with toileting. In these circumstances caregivers must be fully inducted in the use of PPE and hand hygiene [11]. Some of the personal protective equipment utilized as non- pharmaceutical/chemical intervention in preventing COVID-19 infections are discussed below

\subsubsection{Face masks}

It has already been well established that wearing face coverings in public is the most useful personal preventive measures [12]. A group of chemists led by Molina have determined the transmission pathways of COVID-19 by investigating the tendency and mitigation measures in Wuhan, Italy, and New York City, the three major epicenters in the period of January to May, 2020 [13]. Their analysis showed that the airborne transmission route is extremely infectious and the main route for spreading COVID-19. The results concluded that the difference between wearing and not wearing a face mask signifies the main element to restrict the trends of the pandemic by reducing the number of infections. In order to prevent inter-human transmission, the wearing of face covering in public domain is the most useful measure, compared to concurrent social distancing, quarantine as well as contact tracing.

A common surgical or N95 mask was not recommended to use for multiple times until recently. It was unable to selfsterilize for reuse and this caused a high economic cost for a single mask. Moreover, shortage of masks was experienced at the early stage of the pandemic. Hence, there is a need for proper methods to decontaminate the masks in order to extend the life of available supply. The decontamination process should not compromise the proper fit and function of masks [14]. In 2009, a group of researchers reported decontamination methods where a single treatment with hydrogen peroxide vapor, UV light or dry heat below $100^{\circ} \mathrm{C}$ did not affect the ability of $\mathrm{N} 95$ mask to filter small particles. In the context of the current pandemic by COVID-19, there have been several more recent investigations. Recently Schwartz and coworkers in 2020 developed a procedure for sterilizing $\mathrm{N} 95$ masks using hydrogen peroxide vapor. The technique does not alter the fit of the mask and it leaves no residue other than water as a byproduct in the process of decontamination. Although their study did not use SARS-CoV-2, but instead tested with other biological indicators. The study suggests that most of the masks could undergo this decontamination process at least 30 cycles without loss of any fit [15]. Several methods to disinfect $\mathrm{N} 95$ masks was examined [16] and it was discovered that heating $\left(\leq 85^{\circ} \mathrm{C}\right)$ under severe humidity was the most encouraging, non-destructive method for decontamination without altering filtration ability of N95-mask. They initially started by heating the mask at a temperature of $75^{\circ} \mathrm{C}$ because of the wide availability of $75^{\circ} \mathrm{C}$ blanket warming temperature oven in hospitals and later further used $85^{\circ} \mathrm{C}$, and found that $75^{\circ} \mathrm{C}$ was not sufficient to inactivate SARS-CoV-2. The optimized condition for decontamination at $85{ }^{\circ} \mathrm{C}$ with $30 \%$ relative humidity was able to continue up to 50 cycles without compromising the function of the mask. Notably, alcohol and chlorine based disinfectants such as bleach interfere with the electrostatic charge on the masks, which is an important component of the filter and significantly reduce their filtering efficiency. Although, these are good candidates for sanitizing hard surfaces, they should not be used to clean N95 masks. 
Recently, a group of material chemists developed an exotic method to functionalize surgical masks with self-cleaning properties and hence these commercially available masks could be reusable and recyclable where they deposited a few layers of graphene on the surface of the mask [17]. The super-hydrophobic graphene surface does not allow incoming aqueous droplets of virus to stick and more importantly, the surface temperature of this mask can rapidly increase to approximately $80^{\circ} \mathrm{C}$ under the exposure to sunlight, which endows the masks to be reusable just after sunlight sterilization and such mask can give better protection against SARS-CoV-2. Recently, a company, IST also developed a similar mask by coating nanofilms that create a hydrophobic molecular barrier which prevents the absorption of droplets into protective face masks [18].

Apart from blocking the virus droplets by wearing mask, it would be useful to deactivate the fluid droplets of viruses while passing through the mask. For this deactivation, antiviral or mild sanitizing molecules can be placed within the mask so that virions get deactivated when they pass through the mask. However, this strategy of on-mask chemical modulation has to be planned in such a way that the sanitizing molecules should only be released from the mask during exhalation [19].

Overall, physical treatments like UV irradiation, heating and dehydration are useful for deactivation of the virus. This can also be done by chemical sanitization using mild acids, mild oxidants, alcohols, or surfactants [20] [21]. Such chemical approaches can be very effective to restrict the spread and transmission of the virus.

\subsubsection{Hand Gloves}

Hand gloves are inserted into the hands to protect infections due to exposure to blood, bodily fluids, saliva and nasal secretions/droplets or discharges and when handling contaminated equipment. Gloves are equally worn when caregivers are attending to the suspected COVID -19 patients. Increased awareness among healthcare workers of the potential for gloves to provide protection against various pathogenic microorganisms such as SARS-CoV-2 has led to the increased use of gloves in health care in this present COVID -19 pandemic. However, gloves can sometimes be used inappropriately and failure to remove them at the correct time and complete hand hygiene effectively can increase the risk of transmitting infections to vulnerable patients [11]. Each healthcare worker should understand the rationale for glove use and assess the potential risk that each task carries. Gloves do not replace the requirement for good hand hygiene and should be changed when they have become contaminated i.e. between patients and between different procedures on the same or different patients. Hands should always be decontaminated following the removal of gloves. Hand gloves are of several types which includes polythene gloves which are thin and have the tendency to tear. They are not an appropriate choice for healthcare settings. Vinyl gloves have been shown to be less effective than latex gloves in providing an impermeable barrier against SARS-CoV-2. They have loose fitting and unsuitable for procedures that require manual dexterity. The use of vinyl gloves in healthcare is limited [22] [11]. Latex glove such as DPNRL (De Proteinised Natural Rubber Latex) have closer fitting than vinyl gloves and provide a more effective barrier against microorganisms such as SARS-CoV-2 amongst other [22]. They should be non-powdered and have the lowest level of extractable protein available. Healthcare workers who are suspected to be allergic to latex should not wear latex gloves. Similarly, when caring for a patient who is known to be sensitive to latex, an alternative glove should also be available for use. Most healthcare settings are now becoming latex free due to the allergy risk to patients and staff [22]. Nitrile (acrylonitrile) gloves are comparable to natural rubber and are the common choice of gloves for use where a latex free environment is required. There are however reports by some healthcare workers who have also developed allergic reactions to nitrile. Neoprene gloves known to have similar properties to natural rubber latex and are often popular replacement in situations when a latex-free glove is required and manual dexterity have important usage in surgery, however, they are more expensive alternative to natural rubber latex gloves. Disposable plastic/nylon, aprons/gowns which are made from polymer are designed to protect uniforms and clothing from moisture and soiling during direct patient care. In the majority of cases, plastic aprons will be appropriate for standard precautions. In some cases, where extensive contamination of blood and body fluids is anticipated e.g., maternity units, emergency department units or when the patient requires a significant amount of direct care with close skin-to-skin contact, a long sleeved fluid repellent gowns may be more appropriate [23]. Gowns do not need to be sterile unless used for an aseptic procedure such as central line insertion or in an operating theatre. Eye protection, visors or full-face protection and shield which are chemical products are worn to prevent infection as a result of splashing body fluids onto mucous membranes of the nose and also into the eyes. Eyes can be protected by wearing either goggles or a visor. Personal glasses are not a suitable substitute. If reusable eye and face protection is used, it should be decontaminated in accordance with the manufacturer's guidelines. Hands should always be decontaminated after removing the equipment. Surgical masks (Fluid Repellent) equipment provides barrier protection to the wearer from splashes and droplets to the area of the wearer's nose, mouth and respiratory tract. They do not provide protection against aerosolised particles and are not classified as respiratory protective equipment [22 [24]. The purpose of respiratory protection such as filtering face piece (FFP3) respirator mask is to protect the wearer from pathogens spread by the airborne route such as SARS-Co-2 
amongst others and when performing aerosol generating procedures on patients with suspected or known respiratory tract infections [23].

\section{Non-Pharmaceutical Chemically Based Substances}

These are chemically based substances which have been effective in preventing the spread of COVID-19. Some of these substances are soaps and detergents, hand sanitizers, bleach and hydrogen peroxide.

\subsection{Chemistry of Soaps and Detergents in killing SARS-CoV-2}

Soaps and detergents are extremely effective in killing the viruses. Both soaps and detergents chemically contain surfactants (short form for surface-active agents). Surfactant molecules are basically sodium salt of long-chain fatty acid (for instance $\mathrm{C} 17 \mathrm{H} 35 \mathrm{COONa}$, sodium stearate) known as amphiphile. Amphiphile has two parts, fat-like long-chain called hydrophobic tail that avoids water and COO-Na+ end, called hydrophilic (or lyophobic) head. The normal cleaning mechanism of detergents is as follows. When soap or detergent is dissolved in water, many detergent molecules are arranged together forming tiny bubbles called micelle where the hydrophilic head groups are oriented outward facing the water whereas hydrophobic or lipophilic tails are hidden inside forming a hydrophobic pocket that potentially traps normal dirt from our skin or garments [25] Thereafter, these amphiphiles are similar in structure to the lipid molecules of the biological membrane to the extent that surfactant molecules compete with the lipids in the virus membrane and replace them easily, because the virus is a self-assembled structure where the weakest connection is in the lipid bilayer. The attached surfactant molecule (at the membrane) bonds to water at one end and bonds to lipid at the other end resulting in push-pull interactions that breaks the virus membrane. In this way, soap breaks the fat membrane and hence the virus structurally tear or cut apart and get destroyed. Similar to dirt, the constituents of the virus gets washed away with water, as the micelle formed by the soap molecules in the water forms a hydrophobic pocket that traps the viral fragments. Apart from breaking the virus structure, the other role of soap or detergent is that viruses cannot stay stuck to the skin in the presence of soap or detergent, and are thus washed away with water flow [25]. In order for these reactions to be effective, it is recommended that hand washing using soaps or detergents with water should last for at least twenty seconds.

\subsection{Hand sanitizers}

Hand sanitizers are also very useful in getting rid of the virus. It is always recommended to carry a hand sanitizer when someone goes outside the home where soap and water is not accessible or handy. How does hand sanitizers kill the SARS-CoV-2? Chemistry can explain this as well. First and foremost, the sanitizers which are being used to inactivate the SARS-CoV-2 are alcohol based hand sanitizers. According to formulation provided by World Health Organisation, a hand sanitizer basically contains four components: (a) alcohol that can be either ethyl alcohol or isopropanol, (b) water, (c) glycerol and (d) hydrogen peroxide. The selection criteria for ethanol or isopropanol in the manufacture of handsanitizer are presumably due to their good water solubility and non-toxicity. The alcohol is mainly responsible for destroying the virus. This is because lipid bilayer of the virus membrane cannot survive in the presence of alcohol that dissolves the lipid molecules in it and hence the virus membrane melts and virus gets inactivated. Moreover, alcohol causes denaturation of viral proteins where the folded proteins are converted to unfolded proteins leading to loss of their biological activities [26]. Thus, alcohol plays an important role in destroying the virus by melting the lipid membrane and denaturing the protein of the virus. It can be noted that alcohol particularly in the range of 60-90\% is rapidly virucidal. When the concentration of alcohol is below $50 \%$, effectiveness for disinfection is reduced abruptly. Moreover, a higher concentration of alcohol does not necessarily generate more effectiveness. Increasing alcohol percentage concomitantly reduces the percentage of water in sanitizer, while water content also has an important function in destroying viruses. In particular, water is essential as it acts as a catalyst for denaturing the proteins of the cell walls. For instance, $70 \%$ isopropyl alcoholic solutions penetrate the cell membrane efficiently which diffuses the entire cell, denature all proteins, and hence the virus gets destroyed. Significant water content in solution slows down the evaporation process, leading to increase in effectiveness by higher surface contact time. When isopropyl alcohol concentration goes over $91 \%$, it coagulates proteins instantly and as a result, a protective coating is developed which guards other proteins from further coagulation [27] The third component is glycerol acts as a moisturizing agent preventing skin drying out and the fourth component, hydrogen peroxide is generally included to prevent bacterial contamination into the hand sanitizer. Moreover, hydrogen peroxide can assist in destroying virus proteins and genetic materials by its oxidizing property, where this is reduced to water. It can be mentioned that chemical processes are the basis for the production of these materials including hand sanitizers. Apart from sanitizing our hands, hand sanitizers can also be applied on hard/fomite surfaces [27].

Hand sanitizers are portable and hence provide an advantage over soap water, which are not accessible or handy at all places. But in the context of destroying the virus, soapy water is more effective than the sanitizer [28]. Use of large 
amount of sanitizer is certainly not a pragmatic choice as we need to restrict the amount of single time use to a few ml in volume. Moreover, extreme volatility of alcohol is a major setback as the alcohol based sanitizer evaporates very quickly and leaves only a little contact time with virus. Within the limited time of evaporation, the sanitizer may not effectively react and inactivate the viruses especially those reside inside our palm lines. Consequently, the rubbing of both hands after sanitizer application becomes less effective at least to some extents. On contrary, cleaning our hands with soap solution for a minimum of 20 seconds followed by large amount of water ensure the complete exposure of viruses for inactivation. Soap solutions are neither volatile nor expensive as alcohol based sanitizers. Thus the cost to performance ratio clearly gives an advantage to the use of soap solution over alcohol based sanitizers [28].

\subsection{Bleach for surface sanitization}

Fomite surfaces are generally sanitized by disinfectant sprays, for instance, chlorine-based bleach, which is an aqueous solution of sodium hypochlorite ( $\mathrm{NaClO}$ ). The chlorine based bleach is commonly used for household disinfection and cleaning [9]. $\mathrm{NaClO}$ is unstable in solution and relatively stable as dilute conditions that have solvated $\mathrm{Na}+$ and $\mathrm{ClO}-$ ions and this stoichiometric solution is alkaline in nature with $\mathrm{pH} \geq 11$. As a result of this, hypochlorous acid is a weak acid whereas $\mathrm{NaOH}$ is a strong base as given in the following chemical equations.

$$
\mathrm{NaOCl}_{(\mathrm{s})}+\mathrm{H}_{2} \mathrm{O}_{(\mathrm{l})} \leftarrow \rightarrow \mathrm{HOCl}_{(\mathrm{aq})}+\mathrm{NaOH}_{(\mathrm{aq})}
$$

The following species are formed in solutions.

$$
\begin{gathered}
\mathrm{HOCl}_{(\mathrm{aq})} \leftarrow \rightarrow \mathrm{H}^{+}+\mathrm{ClO}_{-(\mathrm{aq})} \\
\mathrm{HOCl}_{(\mathrm{aq})}+\mathrm{Cl}^{-}+\mathrm{H}^{+} \leftarrow \rightarrow \mathrm{Cl}_{2(\mathrm{aq})}+\mathrm{H}_{2} \mathrm{O}
\end{gathered}
$$

Hypochlorite shows a broad range of antimicrobial activity and is useful to kill a number of common pathogens at different concentrations. For instance, hypochlorite is used against rotavirus at the concentration of $0.05 \%$, whereas a higher concentration of $0.5 \%$ is needed for some highly resistant pathogens, for example, C. difficile [29]. In the context of COVID-19, hypochlorite concentration of $0.1 \%$ is recommended [30]. Bleach oxidizes and destroys virus proteins and genetic materials, as $\mathrm{NaClO}$ is unstable in solution and easily decomposes to chlorine. The surfaces should be exposed to hypochlorite solution for at least 10 minutes for killing the viruses. Unlike soap water or hand sanitizers, it can only be applied on hard fomite surfaces, not on our hands. Hypochlorite can be used for washing hands only when the concentration of hypochlorite is very low, at about $0.05 \%$, and this hypochlorite solution is usually prepared with calcium hypochlorite [27]. In order to achieve these final desired concentrations of hypochlorite, it is necessary to dilute the commercially available chlorine-based bleach products where the chlorine concentrations usually vary between $4 \%$ and $6 \%$ [9] [31]. It can be noted that high concentrations of chlorine in commercial bleach can cause corrosion of metal, alloy, many thermoplastic and irritation of skin with potential side-effects.

\subsection{Hydrogen Peroxide for Surface Sanitization}

Hydrogen peroxide solution can only be applied on hard and fomite surfaces, not on our hands and the minimum concentration should be $0.5 \%$ [9]. It oxidizes and destroys virus proteins and genetic materials which should be left on the surfaces for at least 10 minutes for effective killing of viruses. Hydrogen peroxide oxidizes the proteins and RNA of the viruses. Protein cyst residue contains thiol group and it is oxidized to disulphide and other analogues like sulfenic acid, and finally to sulfonic acid by hydrogen peroxide [32]. Moreover, the guanosine unit of the genome is oxidized to 8-oxo-guanosine [33]. However, the use of these chemical disinfectants or sanitization materials is restricted, as it is unable to cover all sorts of exposed areas. In practical application, sanitizers may not act uniformly across the entire surfaces due to concerns regarding volatility and de-wetting. Moreover, it requires repeated application of sanitization periodically to keep the surface virus free. Therefore, it is necessary to develop self-sanitizing surfaces that would gently release disinfecting molecules to clean the surface and thus diminish transmission through objects. It can be noted that such coatings should be non-toxic, long-lasting, and resilient against rubbing and washing. However, material chemists can contribute to this new research area to combat COVID-19.

\section{Conclusion}

The chemistry and the chemical science preventive approach to fight COVID-19 pandemic cannot be swept under the carpet. Emphasis has been given on the use of PPE like facemasks, face masks and washing of hands with soap and water frequently, hand sanitizers, bleach and hydrogen peroxide for surface sanitization has been effective ways of not contracting this ravaging virus. Micelle formation by soap water, destruction of viral membrane by surfactant, oxidation 
of protein by disinfectants, denaturation of protein by alcohol are depicted as part of the mode of action of chemical preventive measures. Even though with the success in the invention of COVID-19 vaccines such as Johnson vaccines, Pfizer vaccines, Astra Zeneca amongst others, there is need to consider the preventive line of action than the curative measures where chemical sciences intervention has contributed in conceptualizing and developing the products for chemical preventive measures whose mechanism are well explained by chemists.

\section{Compliance with ethical standards}

\section{Acknowledgments}

Acknowledgement is extended to the last author for taking time to source for some information needed to get the manuscript ready.

\section{Disclosure of conflict of interest}

The authors declare that they have no competing interest.

\section{References}

[1] Huang C, Wang Y, Li, X, Ren. L, Zhao, J, Hu, Y. et al .Clinical Features of Patients Infected with 2019 Novel Coronavirus in Wuhan, China. Lancet. 2005;395(10223): 497-506.

[2] Velavan T.P, and Meyer C.G. The COVID-19 Epidemic. Trop Med Int Health. 2020; 25(3): 278-280

[3] Phan T. Why-is-70-isopropyl-alcohol-a-better disinfectant-than-99-isopropanol-and-what-is-isopropyl alcoholused-for. Infect. Genet. Evol. 2020;81: 104260.

[4] Sun F., Ganguli A., Nguyen J., Brisbin R., Shanmugam K., Hirschberg D. L., Wheeler M. B.,Bashir R., Nash D. M., Cunningham B. T., Lab Chip , The science of soap-here's how it kills the coronavirus 2020; 20, 1621-1627.

[5] Lu R., Zhao X., Li J., Niu P., Yang B., Wu H .Genomic Characterisation and Epidemiology of 2019 Novel Coronavirus: Implications for Virus Origins and Receptor Binding. Lancet. 2020;395 (10224): 565-574.

[6] De Wit E., Van Doremalen N., Falzarano D., Munster V. J. SARS and MERS: Recent Insights into Emerging Coronaviruses. Nat. Rev. Microbiol. . (2016); 14,523-534.

[7] Zhu N, Zhang D, Wang W, Li, X, Yang, B, Song, J et al. A Novel Coronavirus from Patients with Pneumonia in China. N. Engl. J. Med. 2020;382, 727-733.

[8] Wrapp D, Wang N, Corbett K.S, Goldsmith J.A, Hsieh C, Abiona O. Cryo-EM Structure of the 2019-nCoV Spike in the Perfusion Conformation. Science. 2020;367(6483): 1260-1263.

[9] CDESCC: Infection Prevention and Control. A Publication on the Cleaning and Disinfection of Environmental Surfaces in the Context of COVID-19. (2002). Retrieved on 12/02/2022.

[10] Wigginton K. R., Pecson B. M., Sigstam T., Bosshard F., and Kohn T., WHO (2012): Guide to Local Production: WHOrecommended Handrub Formulations. Environ. Sci. Technol2012; 46, 12069-12078.

[11] Healthy Safety Environment (HSE) on Personal Protective Equipment at work 2nd ed. HSE Books, Norwich, 2005.. Pp 32-34.

[12] Leung C.C, Lam T.H, Cheng K.K. Mass Masking in the COVID-19 Epidemic. People Needs Guidance. Lancet. 2020; 395(10228): 945-947.

[13] Zhang R, Li, Y, Zhang A.L, Wang Y, Molina M.J. Identifying Airborne Transmission as the Dominant Route for the Spread of COVID-19. Proc. Natl. Acad. Sci. 2020; 117(26):14857-14863.

[14] COVID-19 Pandemic Update 15: Can we Disinfect and Reuse N95 Masks? https://www.youtube.com/watch?v=FGEd3LVUFVU. Retrieved on 12/02/2022.

[15] Schwartz A., Stiegell M., Greeson N., Vogel A., Thomann W., Brown M., Sempowski G. D., Alderman T. S., Condreay J. P., Burch J., Wolfe C., Smith B., Lewis S., Applied Biosafety: Journal of ABSA International, 2020;25, 67-70.

[16] Liao L, Xiao W, Zhao M, Yu, X, Wang H, Wang Q .Can N95 Respirators be Reused after Disinfection? How Many Times? ACS Nano. 2020;14(5): 6348-6356. 
[17] Zhong H, Zhu Z, Lin J, Cheung C.F, Lu,V.L, Yan, F. Reusable and Recyclable Graphene Masks with Outstanding Superhydrophobic and Photothermal Performances ACS Nano. 2020;14(5): 6213-6221.

[18] www.insurftech.com

[19] Huang H., Fan C, Li, M, Nie, H.L, Wang. F.B, Wang, H et al. A Call for Physical Scientists and Engineers. ACS Nano, 2020;14(4): 3747-3754.

[20] Wigginton K.R, Ye, Y, Ellenberg R.M. Emerging Investigators Series: The Source and Fate of Pandemic Viruses in the Urban Water Cycle. Environ Sci: Water Res Technol. 2015;1: 735 -746.

[21] Kampf G, Todt D, Pfaender S, Steinmann E., Persistence of Coronaviruses on Inanimate Surfaces and their Iactivation with Biocidal Agents. J. Hosp. Infect. 2020;104(3): 246-251.

[22] Standard Infection Control Precautions Literature Review. Published by Health Protection Board Scotland (2015) available at http://www.hps.scot.nhs.uk/haiic/ic/index.aspx

[23] Coia J.E. Ritchie L., Adisesh A. Makison-Booth, C. Bradley, C. Bunyan .Guidance on the Use of Respiratory and Facial Protection Equipment. Journal of Hospital Infection. 2013; 85: 170-182.

[24] Fraise A., Bradley C. (2009). Ayliffe's Control of Healthcare-Associated Infection: APractical Handbook Fifth Ed. CRC Press, Croydon. 2009; 4-11.

[25] www.scitech.org.au. Retrieved on12/02/2022.

[26] Simmone, A. Hand Hygiene and Hand Sanitizers. A Series from the Family Youth and Community Sciences Department, Florida Cooperative Extension Service, Institute of Food and Agricultural Sciences, University of Florida, Publication no. FCS8788; March 2011. 18-22.

[27] Singh P, Potlia I, Malhotra, S, Dubey, H, Chauhan H. Hand Sanitizer an Alternative to Hand Washing - A Review of Literature. Journal of Advanced Oral Research 2020;1(2): 137-142.

[28] Alex R., Pall T. Which is Better: Sanitizer?https://www.youtube.com/watch?v=x7KKkElpyKQ\&feature=youtube. 2020; Pp 65-68.

[29] Köhler A. T, Rodloff A.C, Labahn M, Reinhardt M, Truyen, U, Speck S.. Efficacy of Sodium Hypochlorite against Multidrug-Resistant Gram Negative Bacteria. J. Hosp. Infect. 2018;100(3): 40-46.

[30] Centers for Disease Control and Prevention(CDC): For General Healthcare Settings in West Africa: How to Prepare and Use Chlorine Solutions. Ebola Hemorrhagic Fever. 2020; 12-20.

[31] Yates T., Allen J., Joseph, and M. L.Lantagne, WASH Interventions in Disease Outbreak Response. Oxfam; Feinstein International Center; UKAID. 2007; 202-212

[32] Van Bergen L. A. H., Roos, G and De Proft, F. From Thiol to Sulfonic Acid: Modelling the Oxidation Pathway of Protein Thiols by Hydrogen Peroxide. J. Phys. Chem. A 2014; 118 (31): 6078-6084.

[33] Hofer T., Badouard C., Bajak E., Ravanat J.-L., Mattsson Å., Cotgreave I. A. Hy drogen Peroxide Causes Greater Oxidation in Cellular RNA than in DNA. Biol. Chem. 2005; 386(4): 333-337. 